\title{
Nondifferentiable Multiobjective Programming with Equality and Inequality Constraints
}

\author{
Iqbal Husain, Vikas K. Jain \\ Department of Mathematics, Jaypee University of Engineering and Technology, Guna, India \\ Email: ihusain11@yahoo.com, jainvikas13@yahoo.com
}

Received February 10, 2013; revised March 18, 2013; accepted March 31, 2013

Copyright (c) 2013 Iqbal Husain, Vikas K. Jain. This is an open access article distributed under the Creative Commons Attribution License, which permits unrestricted use, distribution, and reproduction in any medium, provided the original work is properly cited.

\begin{abstract}
In this paper, we derive optimality conditions for a nondifferentiable multiobjective programming problem containing a certain square root of a quadratic form in each component of the objective function in the presence of equality and inequality constraints. As an application of Karush-Kuhn-Tucker type optimality conditions, a Mond-Weir type dual to this problem is formulated and various duality results are established under generalized invexity assumptions. Finally, a special case is deduced from our result.
\end{abstract}

Keywords: Nondifferentiable Multiobjective Programming Problem; Efficient Solution; Generalized Invexity; Duality; Nondifferentiable Multiobjective Programming Problems with Equality and Inequality

\section{Introduction}

A number of researchers have discussed optimality and duality for a class of nondifferentiable problem containing the square root of a positive semi-definite quadratic form. Mond [1] presented Wolfe type duality while Chandra et al. [2] investigated Mond-Weir type duality for this class of problems. Later, Zhang and Mond [3] validated various duality results for the problem under generalized invexity conditions, it is observed that the popularity of this kind of problems seems to originate from the fact that, even through the objective functions, and/or constraint function are non-smooth, a simple and elegant representation for the dual to this type of problems may be obtained. Obviously non-smooth mathematical programming with more general type functions by means of generalized sub differentials. However, the square root of positive semi-definite quadratic form is one of some of a nondifferentiable function for which sub differentials can be explicitly be written.

Multiobjective optimization problems have been applied in various field of science, where optimal decisions need to be taken in the presence of trade-offs between two or more conflicting objectives. Researchers study multiobjective optimization problems from different viewpoints and, then there exist different goals when setting and solving them. The goal may be finding a representation set of Pareto optimal solutions, and/or qualifying the trade-offs in satisfying the different objectives, and/or finding a single solution that satisfies the preferences of a human decisions making. Motivated with these observations, there has been an increasing interest in studying optimality and duality for nondifferentiable multiobjective programming problems. Duality results for nondifferentiable multiobjective programming problems with square root term appearing in each component of the vector objective derived by Lal et al. [4]. In nondifferentiable multiobjective programming problems, having a support function in each component of the vector objective, further developments for duality results are found in Kim et al. [5] and Yang et al. [6].

In this paper, we obtain optimality conditions for a class of nondifferentiable multiobjective programming problems with equality and inequality involving a square root terms in each component of the objective. For this class of problems, Mond-Weir type dual is formulated and usual duality results are obtained. In the end a special case is generated.

\section{Related Pre-Requisites and Expression of the Problem}

In [1], the following problem is considered:

$$
\begin{array}{cl}
\text { Problem (EP): Minimize } & f(x)+\left(x^{T} B x\right)^{1 / 2} \\
\text { subject to } & g(x) \leq 0 \\
& h(x)=0
\end{array}
$$


where

1) $f: R^{n} \rightarrow R, \quad g: R^{n} \rightarrow R^{m}$ and $h: R^{n} \rightarrow R^{p}$ are continuously differentiable.

2) $B$ is an $n \times n$ symmetric positive semi definite matrix.

The following generalized Schwartz inequality [7] will be needed in the present analysis:

$$
x^{T} B w \leq\left(x^{T} B x\right)^{1 / 2}\left(w^{T} B w\right)^{1 / 2}
$$

The equality in the above holds if, for $\lambda \geq 0$, $B x=\lambda B w$.

The function $\phi(x)=\left(x^{T} B x\right)^{1 / 2}$, being convex and everywhere finite, has a subdifferential in the sense of convex analysis. The subdifferential of $\left(x^{T} B x\right)^{1 / 2}$ is given by

$$
\begin{aligned}
& \partial\left(x^{T} B x\right)^{1 / 2} \\
& =\left\{B w \mid x^{T} B w=\left(x^{T} B x\right)^{1 / 2}, \text { where } w \in R^{n}, \text { and } w^{T} B w \leq 1\right\}
\end{aligned}
$$

We also require the Mangasarian-Fromovitz constraint qualification which is described as the following:

Let $\bar{x} \in \Omega$ be the set of feasible solution of the problem (EP), that is,

$$
\Omega=\left\{x \in R^{n} \mid g(x) \leq 0, h(x)=0\right\}
$$

and by $A(\bar{x})$, the set of inequality active constraint indices, that is,

$$
A(\bar{x})=\left\{j \mid g_{j}(\bar{x})=0\right\},
$$

where $\bar{x} \in \Omega$. We say the Mangasarian-Fromovitz constraint qualification holds at $\bar{x} \in \Omega$ when the equality constraint gradients $\nabla h_{1}(\bar{x}), \nabla h_{2}(\bar{x}), \cdots, \nabla h_{p}(\bar{x})$ are linearly independent and there exist a vector $d \in R^{n}$ such that

$$
\nabla h(\bar{x}) d=0 \text { and } \nabla g_{j}(\bar{x}) d<0 \text {, for all } j \in A(\bar{x}) .
$$

The following theorems (Theorem 2.1 and Theorem 2.2) give Fritz John and Karush-Kuhn-Tucker type optimality conditions using the concept of sub differential obtained by Husain and Srivastav [8] using the concept of subdifferential:

Theorem 2.1 (Fritz John Optimality Conditions): If $\bar{x}$ is an optimal solution of (EP), then there exist Lagrange multipliers $\tau \in R, \quad y \in R^{m}, \quad z \in R^{p}, \quad w \in R^{n}$ such that

$$
\begin{gathered}
\tau(\nabla f(\bar{x})+B w)+\nabla y^{T} g(\bar{x})+\nabla z^{T} h(\bar{x})=0 \\
\bar{x}^{T} B w=\left(\bar{x}^{T} B \bar{x}\right)^{1 / 2} \\
y^{T} g(\bar{x})=0
\end{gathered}
$$

$$
\begin{gathered}
w^{T} B w \leq 1 \\
(\tau, y) \geq 0 \\
(\tau, y, z) \neq 0 .
\end{gathered}
$$

If Mangasarian-Fromovitz constrain qualification (MFCQ) holds at $\bar{x}$, then the above theorem reduces to the following theorem giving Karush-Kuhn-Tucker optimality conditions:

Theorem 2.2 (Karush-Kuhn-Tucker optimality conditions): If $\bar{x}$ is an optimal solution of (EP) and MFCQ holds at $\bar{x}$, then there exist $y \in R^{m}, \quad z \in R^{p}, \quad w \in R^{n}$ such that

$$
\begin{gathered}
\nabla f(\bar{x})+B w+\nabla y^{T} g(\bar{x})+\nabla z^{T} h(\bar{x})=0 \\
\bar{x}^{T} B w=\left(\bar{x}^{T} B \bar{x}\right)^{1 / 2} \\
w^{T} B w \leq 1 \\
y^{T} g(\bar{x})=0 \\
y \geq 0 .
\end{gathered}
$$

The following conventions for inequalities will be used in the subsequent analysis: If $a, b \in R^{n}$, then

$$
\begin{aligned}
& a \geq b \Rightarrow a_{i} \geq b_{i}, i=1,2, \cdots, n \\
& a \geq b \Rightarrow a \geq b \text { and } a \neq b \\
& a>b \Rightarrow a_{i}>b_{i}, i=1,2, \cdots, n .
\end{aligned}
$$

Consider the following multiobjective programming problem containing square root of a certain quadratic form in each component of the objective.

(VEP): Minimize

$$
\left(f_{1}(x)+\left(x^{T} B_{1} x\right)^{1 / 2}, \cdots, f_{k}(x)+\left(x^{T} B_{k} x\right)^{1 / 2}\right)
$$

Subject to

$$
\begin{aligned}
& g(x) \leq 0 \\
& h(x)=0
\end{aligned}
$$

where $f, g$ and $h$ are the same as in (EP).

$$
\text { Let } \Omega=\left\{x \in R^{n} \mid g(x) \leq 0, h(x)=0\right\}
$$

Definition 2.1 A point $\bar{x} \in \Omega$ is said to be an efficient solution of (EP) if there exists no $x \in \Omega$ such that

$$
f_{r}(x)+\left(x^{T} B_{r} x\right)^{1 / 2}<f_{r}(\bar{x})+\left(\bar{x}^{T} B_{r} \bar{x}\right)^{1 / 2},
$$

for some $r \in K=\{1,2, \cdots, k\}$ and

$$
f_{i}(x)+\left(x^{T} B_{i} x\right)^{1 / 2} \leqq f_{i}(\bar{x})+\left(\bar{x}^{T} B_{i} \bar{x}\right)^{1 / 2},
$$

for $i \in K_{r}=K-r$.

The following results relate an efficient solution of 
(EP) of $k$-scalar objective programming problems.

Lemma 2.2 (Chankong and Haimes [9]): A point $\bar{x} \in \Omega$ is an efficient solution of (EP) if and only if $\bar{x}$ is an optimal solution of $\left(E P_{r}\right)$ for each $r \in K$.

$$
\begin{aligned}
& \left(E P_{r}\right) \text { : Minimize } f_{r}(x)+\left(x^{T} B_{r} x\right)^{1 / 2} \\
& \text { subject to } g(x) \leqq 0 \\
& h(x)=0 \\
& f_{i}(x)+\left(x^{T} B_{i} x\right)^{1 / 2}<f_{i}(\bar{x})+\left(\bar{x}^{T} B_{i} \bar{x}\right)^{1 / 2}, i \in K_{r}
\end{aligned}
$$

We recall the following definitions of generalized invexity which will be used to derive various duality results.

Definitions 2.2: 1) A function $\phi: R^{n} \rightarrow R$ is said to be quasi-invex with respect to a vector function $\eta=\eta(x, u)$, if

$$
\phi(x) \leq \phi(u) \Rightarrow \eta^{T}(x, u) \nabla \phi(u) \leq 0
$$

2) A function $\phi$ is said to be pseudo-invex with respect to a vector function $\eta=\eta(x, u)$, if

$$
\eta^{T}(x, u) \nabla \phi(u) \geq 0 \Rightarrow \phi(x) \geq \phi(u) \phi .
$$

3) $\phi$ is said to be the strictly pseudoinvex with respect to $\eta$ if $x \neq u$,

$$
\eta^{T}(x, u) \nabla \phi(u) \geq 0 \Rightarrow \phi(x)>\phi(u) .
$$

Equivalently, if

$$
\phi(x) \leq \phi(u) \Rightarrow \eta^{T}(x, u) \nabla \phi(u)<0 .
$$

\section{Optimality Conditions}

In this section, the optimality conditions for the problem (EP) are obtained.

Theorem 3.1 (Fritz John Type Optimality Conditions): If $\bar{x}$ be an efficient solution of (EP), then there exist $\lambda_{i} \in R$, for $i \in K, y \in R^{m}, z \in R^{p}$ and $w \in R^{n}$ such that

$$
\begin{gathered}
\sum_{i=1}^{k} \lambda_{i}\left(\nabla f_{i}(\bar{x})+B_{i} \bar{x}\right)+\nabla y^{T} g(\bar{x})+\nabla z^{T} h(\bar{x})=0 \\
\bar{x}^{T} B_{i} w_{i}=\left(\bar{x}^{T} B_{i} \bar{x}\right)^{1 / 2}, i \in K \\
w_{i}^{T} B_{i} w_{i} \leqq 1, i \in K \\
y^{T} g(\bar{x})=0 \\
(\lambda, y) \geqq 0 \\
(\lambda, y, z) \neq 0
\end{gathered}
$$

Proof: Since $\bar{x}$ is an efficient solution of (EP), by Lemma $2.1 \bar{x}$ is an optimal solution of $\left(E P_{r}\right)$ for each $r \in K$ and hence in particular of $\left(E P_{1}\right)$. Therefore by Theorem 2.1 there exist $\lambda_{i} \in R$, for $i \in K, y \in R^{m}$, $z \in R^{p}$ and $w \in R^{n}$ such that

$$
\begin{gathered}
\sum_{i=1}^{k} \lambda_{i}\left(\nabla f_{i}(\bar{x})+B_{i} \bar{x}\right)+\nabla y^{T} g(\bar{x})+\nabla z^{T} h(\bar{x})=0 \\
\bar{x}^{T} B_{i} w_{i}=\left(\bar{x}^{T} B_{i} \bar{x}\right)^{1 / 2}, i \in K \\
y^{T} g(\bar{x})=0 \\
w_{i}^{T} B_{i} w_{i} \leq 1, i \in K \\
\left(\lambda_{1}, \lambda_{2}, \cdots, \lambda_{k}, \bar{y}\right) \geq 0 \\
\left(\lambda_{1}, \lambda_{2}, \cdots, \lambda_{k}, \bar{y}, \bar{z}\right) \neq 0
\end{gathered}
$$

The theorem follows.

Theorem 3.2 (Kuhn-Tucker type necessary optimality conditions): If $\bar{x}$ be an optimal solution of (VEP) and let for $r \in K$, the constraints of $\left(E P_{r}\right)$ satisfy MECQ. Then there exist $\lambda \in R^{k}, y \in R^{m}, \quad z \in R^{p}$ and $w \in R^{n}$ such that

$$
\begin{gathered}
\sum_{i=1}^{k} \lambda_{i}\left(\nabla f_{i}(\bar{x})+B_{i} \bar{x}\right)+\nabla y^{T} g(\bar{x})+\nabla z^{T} h(\bar{x})=0 \\
y^{T} g(\bar{x})=0 \\
\bar{x}^{T} B_{i} w_{i}=\left(\bar{x}^{T} B_{i} \bar{x}\right)^{1 / 2}, i=1,2, \cdots, k \\
w_{i}^{T} B_{i} w_{i} \leq 1 \\
\lambda \geq 0 \\
y \geq 0 .
\end{gathered}
$$

Proof: Since $\bar{x}$ is an optimal solution of (VEP), by Lemma 3.1, $\bar{x}$ is an optimal solution of $\left(E P_{r}\right)$ for each r. As for some $r$, the constraint of $\left(E P_{r}\right)$ satisfy MFCQ at $\bar{x}$, by Theorem 2.2 of their exist $0<\lambda_{r} \in R$, $0 \leq \lambda_{i} \in R, i \in K_{r}, y \in R^{m}, z \in R^{p}$ and $w \in R^{n}$ such that

$$
\begin{aligned}
& \lambda_{r}\left(\nabla f_{r}(\bar{x})+B_{r} w_{r}\right)+\sum_{i=1}^{k} \lambda_{i}\left(\nabla f_{i}(\bar{x})+B_{i} w_{i}\right) \\
& +\nabla y^{T} g(\bar{x})+\nabla z^{T} h(\bar{x})=0
\end{aligned}
$$

yielding

$$
\begin{gathered}
\sum_{i=1}^{k} \lambda_{i}\left(\nabla f_{i}(\bar{x})+B_{i} w_{i}\right)+\nabla y^{T} g(\bar{x})+\nabla z^{T} h(\bar{x})=0 \\
y^{T} g(\bar{x})=0 \\
\bar{x}^{T} B_{i} w_{i}=\left(\bar{x} B_{i} \bar{x}\right)^{1 / 2}, i \in K \\
w_{i}^{T} B_{i} w_{i} \leqq 1, i \in K \\
\lambda_{r}>0,0 \leqq \lambda_{i} \in R, i \in K_{r} \\
y \geq 0
\end{gathered}
$$

From the above relation it is obvious that the theorem follows. 
In Theorem 3.2, we assume MFCQ for some $\left(E P_{r}\right)$, which implies $\lambda \geq 0$. In the following theorem, we assume MFCQ for every $\left(E P_{r}\right)$ and obtain $\lambda>0$.

Theorem 3.3 (Kuhn-Tucker type optimality conditions): If $\bar{x}$ be an efficient of (VEP) and let for each $r \in K$, the constraints of $\left(E P_{r}\right)$ satisfy MECQ at $\bar{x}$. Then there exist $\lambda \in R^{k}, y \in R^{m}, z \in R^{p}$ and $w \in R^{n}$ such that

$$
\begin{gathered}
\sum_{i=1}^{k} \lambda_{i}\left(\nabla f_{i}(\bar{x})+B_{i} w_{i}\right)+\nabla y^{T} g(\bar{x})+\nabla z^{T} h(\bar{x})=0 \\
y^{T} g(\bar{x})=0 \\
\left(\bar{x}^{T} B_{i} \bar{x}\right)^{1 / 2}=\bar{x}^{T} B_{i} w_{i}, i \in K \\
w_{i}^{T} B_{i} w_{i} \leqq \\
\lambda>0 \\
\sum_{i=1}^{k} \lambda_{i}=1 \\
y \geqq 0
\end{gathered}
$$

Proof: Since $\bar{x}$ is an efficient of (VEP) by Lemma 3.1, $\bar{x}$ is an optimal solution of $\left(E P_{r}\right)$, by Kuhn-Tucker type necessary optimality conditions, for each $r \in K$, there exist $v_{i}^{r} \in R,\left(i \in K_{r}\right), \mu_{j}^{r} \in R,(j \in M), \quad \delta_{l}^{r} \in R$, $(l \in L)$ and $w \in R^{n}$ such that

$$
\begin{aligned}
& \nabla f_{i}(\bar{x})+B_{i} w_{i}+\sum_{i \in K_{r}} v_{i}^{r}\left(\nabla f_{i}(\bar{x})+B_{i} w_{i}\right) \\
&+\sum_{j=1}^{m} \mu_{j}^{r} \nabla g_{j}(\bar{x})+\sum_{l=1}^{p} \delta_{l}^{r} \nabla h(\bar{x})=0 \\
& \sum_{j=1}^{m} \mu_{j}^{r} g_{j}(\bar{x})=0 \\
& v_{i}^{r} \geqq 0, i \in K_{r} \\
& y \leqq 0
\end{aligned}
$$

Summing over $i \in K$, we get

$$
\begin{aligned}
& \sum_{i=1}^{k}\left(v_{i}^{1}+v_{i}^{2}+\cdots+v_{i}^{k}\right)\left(\nabla f_{i}(\bar{x})+B_{i} w_{i}\right) \\
& +\sum_{j=1}^{m}\left(\mu_{j}^{1}+\mu_{j}^{2}+\cdots+\mu_{j}^{k}\right) \nabla g_{j}(\bar{x}) \\
& +\sum_{l=1}^{p}\left(\delta_{l}^{1}+\delta_{l}^{2}+\cdots+\delta_{l}^{k}\right) \nabla h(\bar{x})=0 \\
& \sum_{j=1}^{m}\left(\mu_{j}^{1}+\mu_{j}^{2}+\cdots+\mu_{j}^{k}\right) g_{j}(\bar{x})=0
\end{aligned}
$$

where $v_{i}^{i}=1$ for $i \in K$

$$
\begin{aligned}
& \sum_{i=1}^{k} v_{i}\left(\nabla f_{i}(\bar{x})+B_{i} w_{i}\right)+\sum_{j=1}^{m} \mu_{j} \nabla g_{j}(\bar{x}) \\
& +\sum_{l=1}^{p} \delta_{l}^{r} \nabla h(\bar{x})=0
\end{aligned}
$$

where $v_{i}=1+\sum_{r \in K_{r}} v_{i}^{r}>0, i \in K, \mu_{j} \in \sum_{r=1}^{k} \mu_{j}^{r} \geq 0, i \in M$

$$
\text { and } \begin{aligned}
& \delta_{l}= \sum_{r=1}^{k} \delta_{l}^{r}, l \in P=\{1,2, \cdots, p\} \\
& \sum_{j=1}^{m} \mu_{j}^{i} g_{j}(\bar{x})=0
\end{aligned}
$$

Dividing throughout the above relation and setting, by $\sum_{i=1}^{k} v_{i}$

$$
\begin{aligned}
& \lambda_{i}=v_{i} / \sum_{i=1}^{k} v_{i}, i \in K, y_{j}=\mu_{j} / \sum_{i=1}^{k} v_{i}, \\
& j \in M, z_{l}=\delta_{l} / \sum_{i=1}^{k} v_{i}, l \in\{1,2, \cdots, p\}
\end{aligned}
$$

We obtain,

$$
\begin{aligned}
\sum_{i=1}^{k} \lambda_{i}\left(\nabla f_{i}(\bar{x})+B^{i} w_{i}\right)+\sum_{j=1}^{m} y_{j} \nabla g_{j}(\bar{x})+\sum_{l=1}^{p} z_{l} \nabla h(\bar{x}) & =0 \\
\sum_{j=1}^{m} y_{j} \nabla g_{j}(\bar{x}) & =0
\end{aligned}
$$

or

$$
\begin{aligned}
& \sum_{i=1}^{k} \lambda_{i}\left(\nabla f_{i}(\bar{x})+B_{i} \bar{x}\right)+\nabla y^{T} g(\bar{x})+\nabla z^{T} h(\bar{x})=0 \\
& y^{T} g(\bar{x})=0 \\
& \lambda>0 \\
& \sum_{i=1}^{k} \lambda_{i}=1 \\
& y \geqq 0 .
\end{aligned}
$$

\section{Mond-Weir Type Duality}

We formulate the following differentiable multiobjective dual nonlinear problem for (VEP):

(M-WED): Maximize

$$
\begin{gathered}
\left(f_{1}(u)+u^{T} B_{1} w, \cdots, f_{k}(u)+u^{T} B_{k} w\right) \\
\text { subject to } \\
\sum_{i=1}^{k} \lambda_{i}\left(\nabla f_{i}(\bar{x})+B_{i} w_{i}\right)+\nabla y^{T} g(u)+\nabla z^{T} h(u)=0 \\
y^{T} g(u) \geq 0 \\
z^{T} h(u) \geq 0 \\
w_{i}^{T} B_{i} w_{i} \stackrel{1}{=} i=1,2, \cdots, k \\
\lambda>0 \\
y \geq 0
\end{gathered}
$$

In the following, we shall use $\Gamma$ for the set of feasible solutions of (M-WED) 
Theorem 4.1 (Weak Duality): Let $x \in \Omega$ and $(u, \lambda, y, z) \in \Gamma$ such that with respect to the same $\eta$,

1) $\sum \lambda_{i}\left(f_{i}()+.(.)^{T} B_{i} w_{i}\right)$ is pseudoinvex

2) $y^{T} g($.$) is quasi-invex, and$

3) $z^{T} h($.$) is quasi-invex.$

Then

$$
\begin{gathered}
f_{r}(x)+\left(x^{T} B_{r} x\right)^{1 / 2}<f_{r}(u)+u^{T} B_{r} w_{r}, \text { for some } r \in K \\
f_{i}(x)+\left(x^{T} B_{i} x\right)^{1 / 2} \leqq f_{i}(u)+u^{T} B_{i} w_{i}, i \in K_{r}
\end{gathered}
$$

cannot hold.

Proof: Suppose the contrary that (9) and (10) hold. Since $\lambda>0$, the above inequalities (9) and (10) give

$$
\begin{aligned}
\sum_{i=1}^{k} \lambda_{i}\left(f_{i}(x)+\left(x^{T} B_{i} x\right)^{1 / 2}\right) & <\sum_{i=1}^{k} \lambda_{i}\left(f_{i}(u)+u^{T} B_{i} w_{i}\right) \\
y^{T} g(x) & \leq y^{T} g(u) \\
z^{T} h(x) & \leq z^{T} h(u)
\end{aligned}
$$

These inequalities because of quasi-invexity of $y^{T} g($.) and $z^{T} h($.$) imply$

$$
\begin{aligned}
& \eta^{T} \nabla y^{T} g(u) \leq 0 \\
& \eta^{T} \nabla z^{T} h(u) \leq 0
\end{aligned}
$$

Combining these, we give

$$
\eta^{T}\left(\nabla y^{T} g(u)+\nabla z^{T} h(u)\right) \leq 0
$$

Using the equality constraint of (M-WED), this yields,

$$
\eta^{T}\left(\sum_{i=1}^{k} \lambda_{i}\left(f_{i}(u)+u^{T} B_{i} w_{i}\right)\right) \geq 0
$$

This, because of 1 ), implies

$$
\sum_{i=1}^{k} \lambda_{i}\left(f_{i}(x)+x^{T} B_{i} w_{i}\right) \geq \sum_{i=1}^{k} \lambda_{i}\left(f_{i}(u)+u^{T} B_{i} w_{i}\right)
$$

Using $x^{T} B_{i} w_{i} \leqq\left(x^{T} B_{i} x\right)^{1 / 2}$ this yields,

$$
\sum_{i=1}^{k} \lambda_{i}\left(f_{i}(x)+\left(x^{T} B_{i} x\right)^{1 / 2}\right) \geqq \sum_{i=1}^{k} \lambda_{i}\left(f_{i}(u)+u^{T} B_{i} w_{i}\right)
$$

Hence the result follows.

Theorem 4.2 (Strong Duality): Let $\bar{x}$ satisfy MFCQ and be an efficient solution (VEP). Then there exist $\lambda \in R^{k}, y \in R^{m}, z \in R^{p}$ and $w \in R^{n}$ such that $(\bar{x}, y, z, \lambda, w)$ is feasible for (M-WED) and the two objective functions are equal. Furthermore, if the weak duality holds for all feasible solution of (VEP) and (M-WED), then $(\bar{x}, y, z, \lambda, w)$ is an efficient solution of the (M-WED).

Proof: Since $\bar{x}$ is an efficient solution (VEP) satisfy
MFCQ, therefore by Theorem (3.3), there exist $\lambda \in R^{k}$, $y \in R^{m}, z \in R^{p}$ and $w_{i} \in R^{n}, i \in K$ satisfy

$$
\begin{aligned}
\sum_{i=1}^{k} \lambda_{i}\left(\nabla f_{i}(\bar{x})+B_{i} w_{i}\right) & +\nabla y^{T} g(\bar{x})+\nabla z^{T} h(\bar{x})=0 \\
y^{T} g(\bar{x}) & =0 \\
\bar{x}^{T} B_{i} w_{i} & =\left(\bar{x}^{T} B_{i} \bar{x}\right)^{1 / 2}, i \in K \\
w_{i}^{T} B_{i} w_{i} \leqq 1 & \\
\lambda & \geq 0 \\
y \geq 0 & \geqq 0
\end{aligned}
$$

Hence $\left(\bar{x}, \lambda, y, z, w_{1}, \cdots, w_{k}\right)$ satisfies the constraints of (M-WED) and

$$
f_{i}(\bar{x})+\left(\bar{x}^{T} B_{i} \bar{x}\right)^{1 / 2}=f_{i}(x)+x^{T} B_{i} w_{i}, i \in K .
$$

i.e. the two objective functions have the same value.

Now we claim that $\left(\bar{x}, \lambda, y, z, w_{1}, \cdots, w_{k}\right)$ is an efficient solution of (M-WED). If not, then there exist $(\hat{u}, \lambda, y, z, w) \in \Gamma$

$$
\begin{aligned}
& \left(f_{1}(\hat{u})+\hat{u} B_{1} w_{1}, \cdots, f_{k}(\hat{u})+\hat{u} B_{k} w_{k}\right) \\
& \geq\left(f_{1}(\bar{x})+\bar{x}^{T} B_{1} w_{1}, \cdots, f_{k}(\bar{x})+\bar{x}^{T} B_{k} w_{k}\right)
\end{aligned}
$$

As $\bar{x}^{T} B_{i} w_{i}=\left(\bar{x}^{T} B_{i} \bar{x}\right)^{1 / 2}, i \in K$, we have

$$
\begin{aligned}
& \left(f_{1}(\hat{u})+\hat{u} B_{1} w_{1}, \cdots, f_{k}(\hat{u})+\hat{u} B_{k} w_{k}\right) \\
& \geq\left(f_{1}(\bar{x})+\left(\bar{x}^{T} B_{1} \bar{x}\right)^{1 / 2}, \cdots, f_{k}(\bar{x})+\left(\bar{x}^{T} B_{k} \bar{x}\right)^{1 / 2}\right)
\end{aligned}
$$

This contradicts Theorem 4.1 Hence $(\bar{x}, y, z, \lambda, w)$ is an efficient solution.

Theorem 4.3 (Strict-converse duality): Let $\bar{x}$ and $(u, \lambda, y, z, w)$ be an efficient solution of (VEP) and (M-WED), such that

$$
\sum_{i=1}^{k} \lambda_{i}\left(f_{i}(\bar{x})+\bar{x}^{T} B_{i} w_{i}\right)=\sum_{i=1}^{k} \lambda_{i}\left(f_{i}(\bar{u})+\bar{u}^{T} B_{i} w_{i}\right)
$$

If with respect to the same $\eta$,

(A $\left.\mathrm{A}_{1}\right) \sum_{i=1}^{k} \lambda_{i}\left(f_{i}()+.(.) B_{i} w_{i}\right)$ is strictly pseudoinvex,

$\left(\mathrm{A}_{2}\right) y^{T} g($.$) is quasi-invex, and$

$\left(\mathrm{A}_{3}\right) z^{T} h($.$) is quasi-invex$ then $\bar{x}=\bar{u}$.

(11)

Proof: Let $\bar{x} \neq \bar{u}$. By hypothesis $\left(\mathrm{A}_{1}\right)$, we have from

$$
\eta^{T} \sum_{i=1}^{k} \lambda_{i}\left(\nabla f_{i}(\bar{u})+B_{i} w_{i}\right)<0
$$

By hypothesis $\left(\mathrm{A}_{2}\right)$ and $\left(\mathrm{A}_{3}\right)$ we have

$$
\eta^{T} \nabla y^{T} g(\bar{u}) \leq 0
$$




$$
\eta^{T} \nabla z^{T} h(\bar{u}) \leqq 0
$$

Combining (12), (13) and (14), we have

$$
\eta^{T}\left[\sum_{i=1}^{k} \lambda_{i}\left(\nabla f_{i}(\bar{u})+B_{i} w_{i}\right)+\nabla y^{T} g(u)+\nabla z^{T} h(u)\right]<0
$$

which contradict the equality constraint of (M-WED). Hence $\bar{x}=\bar{u}$.

\section{Theorem 4.4 (Converse duality):}

Let $\left(\bar{x}, y, \lambda, z, w_{1}, \cdots, w_{k}\right)$ be an efficient solution of (M-WED) at which

1) the matrix $\nabla^{2}\left(\lambda^{T} f(\bar{x})+y^{T} g(\bar{x})+z^{T} h(\bar{x})\right)$ is positive or negative definite and

2) the vectors $\nabla y^{T} g(\bar{x})$ and $\nabla z^{T} h(\bar{x})$ are linearly independent .

If, for all feasible $\left(\lambda, \bar{x}, u, y, z, w_{1}, \cdots, w_{k}\right)$,

$$
\sum_{i=1}^{k} \lambda_{i}\left(f_{i}(.)+(.)^{T} B_{i} w_{i}\right) \text { is pseudoinvex, } y^{T} g(.) \text { is }
$$

qausi-invex and $z^{T} h($.$) is quasi-invex with respect to$ the same $\eta$, then $\bar{x}$ is an efficient solution (EP).

Proof: By Theorem 3.3, there exist $\tau \in R^{k}, \theta \in R^{n}$, $\alpha \in R, \beta \in R, \gamma_{i} \in R, i=1,2, \cdots, k, \xi \in R^{k}$ and $\eta \in R^{m}$ such that

$$
\begin{gathered}
\sum_{i=1}^{k} \tau_{i}\left(\nabla f_{i}(\bar{x})+B_{i} w_{i}\right) \\
+\theta^{T} \nabla^{2}\left(\lambda^{T} f(\bar{x})+y^{T} g(\bar{x})+z^{T} h(\bar{x})\right) \\
+\alpha \nabla y^{T} g(\bar{x})+\beta \nabla z^{T} h(\bar{x})=0 \\
\tau_{i}\left(x^{T} B_{i}\right)+\theta \lambda_{i} \beta_{i}-2 \gamma_{i} B_{i} w_{i}=0 \\
\theta\left(\nabla f_{i}(\bar{x})+B_{i} w_{i}\right)+\xi_{i}=0 \\
\theta \nabla g(\bar{x})+\alpha g(\bar{x})+\eta=0 \\
\theta \nabla h(\bar{x})+\beta h(\bar{x})=0 \\
\alpha y^{T} g(\bar{x})=0 \\
\beta z^{T} h(\bar{x})=0 \\
\eta^{T} y=0 \\
\xi^{T} \lambda=0 \\
\gamma_{i}\left(1-w_{i} B_{i} w_{i}\right)=0, i \in K \\
(\tau, \alpha, \beta, \xi, \gamma, \eta) \geqq 0 \\
(\tau, \alpha, \beta, \theta, \xi, \gamma, \eta) \neq 0
\end{gathered}
$$

Multiplying (15) by $\lambda_{i}$ and summing over $i$, we have

$$
\sum \tau_{i}\left(\sum_{i=1}^{k} \lambda_{i}\left(\nabla f_{i}(\bar{x})+B_{i} w_{i}\right)\right)
$$

$$
\begin{aligned}
& +\left(\sum_{i=1}^{k} \lambda_{i}\right) \theta^{T} \nabla^{2}\left(\lambda^{T} f(\bar{x})+y^{T} g(\bar{x})+z^{T} h(\bar{x})\right) \\
& +\left(\sum_{i=1}^{k} \lambda_{i}\right) \alpha \nabla y^{T} g(\bar{x})+\left(\sum_{i=1}^{k} \lambda_{i}\right) \beta \nabla z^{T} h(\bar{x})=0
\end{aligned}
$$

Using the equality constraint, we have

$$
\begin{gathered}
\left(\sum_{i=1}^{k}\left(\tau_{i}-\alpha \lambda_{i}\right)\right) \nabla y^{T} g(\bar{x})+\left(\sum_{i=1}^{k}\left(\tau_{i}-\alpha \lambda_{i}\right)\right) \nabla z^{T} h(\bar{x}) \\
-\left(\sum_{i=1}^{k} \lambda_{i}\right) \theta^{T} \nabla^{2}\left(\lambda^{T} f(\bar{x})+y^{T} g(\bar{x})+z^{T} h(\bar{x})\right)=0
\end{gathered}
$$

$$
\begin{aligned}
& \left(\sum_{i=1}^{k}\left(\tau_{i}-\alpha \lambda_{i}\right)\right) \theta^{T} \nabla y^{T} g(\bar{x}) \\
& +\left(\sum_{i=1}^{k}\left(\tau_{i}-\alpha \lambda_{i}\right)\right) \theta^{T} \nabla z^{T} h(\bar{x}) \\
& -\left(\sum_{i=1}^{k} \lambda_{i}\right) \theta^{T} \nabla^{2}\left(\lambda^{T} f(\bar{x})+y^{T} g(\bar{x})+z^{T} h(\bar{x})\right) \theta=0
\end{aligned}
$$

From (18) and (19), we have

$$
\begin{aligned}
& \theta^{T} \nabla y^{T} g(\bar{x})=0 \\
& \theta^{T} \nabla z^{T} h(\bar{x})=0
\end{aligned}
$$

Using these in (15), we have

$$
\theta^{T} \nabla^{2}\left(\lambda^{T} f(\bar{x})+y^{T} g(\bar{x})+z^{T} h(\bar{x})\right) \theta=0
$$

which because of the hypothesis 1 ) gives $\theta=0$.

Using $\theta=0$ and the hypothesis 2), we have

$$
\left(\sum_{i=1}^{k}\left(\tau_{i}-\alpha \lambda_{i}\right)\right)=0,\left(\sum_{i=1}^{k}\left(\tau_{i}-\beta \lambda_{i}\right)\right)=0
$$

Let $\tau_{i}=0, i=1,2, \cdots, k$. Then (30) implies $\alpha=0=\beta$. The relations (17) and (18) implies $\eta=0$ and $\xi=0$. Using $\theta=0$ and $\tau=0$ along with (24) in (16), we get $\gamma_{i}=0, i \in K, \theta=0$.

Thus $(\alpha, \lambda, \beta, \eta, \xi, \theta)=0$ a contradiction to (26). Hence $\tau>0$. Consequently $\alpha>0$ and $\beta>0$. Using $\theta=0, \alpha>0$ and $\beta>0$, in (18) and (19), we have

$$
g(\bar{x}) \leq 0, h(\bar{x})=0
$$

This implies that $\bar{x} \in \Omega$.

If $\theta=0$ in (16), we have

$$
B_{i} \bar{x}=\left(\frac{2 \gamma_{i}}{\tau_{i}}\right) B_{i} w_{i}, i \in K
$$

Hence by Schwartz inequality

$$
\bar{x}^{T} B_{i} w_{i}=\left(\bar{x}^{T} B_{i} \bar{x}\right)^{1 / 2}\left(w_{i}^{T} B_{i} w_{i}\right)^{1 / 2}
$$

If $\gamma_{i}>0$, then (24) implies 


$$
w_{i}^{T} B_{i} w_{i} \leqq 1, i \in K
$$

Consequently (32) yields

$$
\bar{x}^{T} B_{i} w_{i}=\left(\bar{x}^{T} B_{i} \bar{x}\right)^{1 / 2}, i \in K
$$

If $\gamma_{i}=0$, then (31) implies $B_{i} \bar{x}=0$. So we still get

$$
\left(\bar{x}^{T} B_{i} \bar{w}_{i}\right)=\left(\bar{x}^{T} B_{i} \bar{x}\right)^{1 / 2} i \in K
$$

Thus by (33), we have

$$
f_{i}(\bar{x})+\left(\bar{x}^{T} B_{i} \bar{x}\right)^{1 / 2}=f_{i}(\bar{x})+\bar{x}^{T} B_{i} \bar{w}_{i}, i \in K .
$$

implying the two objective functions have the same value.

Now, assume that $\bar{x}$ is not an efficient solution of (VEP), then there exists $\hat{x} \in \Omega$ such that

$$
f_{r}(\hat{x})+\left(\hat{x}^{T} B_{r} \hat{x}\right)^{1 / 2}<f_{r}(\bar{x})+\left(\bar{x}^{T} B_{r} \bar{x}\right)^{1 / 2},
$$

for some $r \in K$ and

$$
f_{i}(\hat{x})+\left(\hat{x}^{T} B_{i} \hat{x}\right)^{1 / 2} \leqq f_{i}(\bar{x})+\bar{x}^{T} B_{i} w_{i} \text {, for } i \in K_{r}
$$

Using $\left(\bar{x}^{T} B_{i} \bar{x}\right)^{1 / 2}=\bar{x}^{T} B_{i} \bar{w}_{i}, i \in K$, we have

$$
f_{r}(\hat{x})+\left(\hat{x}^{T} B_{r} \hat{x}\right)^{1 / 2}<f_{r}(\bar{x})+\bar{x}^{T} B_{r} w_{r},
$$

for some $r \in K$

$$
f_{i}(\hat{x})+\left(\hat{x}^{T} B_{i} \hat{x}\right)^{1 / 2} \leqq f_{i}(\bar{x})+\bar{x}^{T} B_{i} w_{i}, \text { for } i \in K_{r}
$$

This contradicts Theorem 4.1. Hence $\bar{x}$ is an efficient solution of (VEP).

\section{A Special Case}

If $K=\{1\}, f_{i}=f$ and $B_{i}=B$, our problems reduce to the following problems recently studied by Husain and Srivastav [8]:

(EP): Minimize $f(x)+\left(x^{T} B x\right)^{1 / 2}$

subject to

$$
\begin{aligned}
& g(x) \leq 0 \\
& h(x)=0
\end{aligned}
$$

(M-WED): Maximize $f(u)+u^{T} B w$

subject to

$$
\begin{aligned}
& \nabla f(u)+B w+\nabla y^{T} g(u)+\nabla z^{T} h(u)=0 \\
& y^{T} g(u) \geq 0 \\
& z^{T} h(u) \geq 0 \\
& w^{T} B w \leq 1, \\
&=0 \\
& y \geq 0
\end{aligned}
$$

\section{Conclusion}

In this research optimality conditions are derived for a nondifferentiable multiobjective programming problem containing a certain square root of a quadratic form in each component of the objective function in the presence of equality and inequality constraints embodying many realistic problems. A Mond-Weir type dual to this problem is formulated and usual duality theorems are proved under appropriate generalized invexity conditions. A special case is also obtained from our duality results. Our results can be revisited in the multiobjective setting of a nondifferentiable control problem.

\section{Acknowledgements}

The authors acknowledge anonymous referees for their valuable comments which have improved the presentation of this research paper.

\section{REFERENCES}

[1] B. Mond, "A Class of Nondifferentiable Mathematical Programming Problems," Journal of Mathematical Analysis and Applications, Vol. 46, No. 1, 1974, pp. 169-174. doi:10.1016/0022-247X(74)90289-3

[2] S. Chandra, B. D. Craven and B. Mond, "Generalized Concavity and Duality with a Square Root Term," Optimization, Vol. 16, No. 5, 1985, pp. 654-662. doi:10.1080/02331938508843062

[3] J. Zhang and B. Mond, "Duality for a Nondifferentiable Programming Problem," Bulletin of the Australian Mathematical Society, Vol. 55, No. 1, 1997, pp. 29-44. doi:10.1017/S0004972700030513

[4] S. N. Lal, B. Nath and A. Kumar, "Duality for Some Nondifferentiable Static Multiobjective Programming Problems," Journal of Mathematical Analysis and Applications, Vol. 186, No. 3, 1994, pp. 862-867. doi:10.1006/jmaa.1994.1337

[5] S. J. Kim, M. H. Kim and D. S. Kim, "Optimality and Duality for a Class of Nondifferentiable Multiobjective Fractional Programming Problems," Journal of Optimization Theory and Applications, Vol. 129, No. 1, 2006, pp. 131-146. doi:10.1007/s10957-006-9048-1

[6] X. M. Yang, K. L. Teo and X. Q. Yang, "Duality for a Class of Nondifferentiable Multiobjective Programming Problem," Journal of Mathematical Analysis and Applications, Vol. 252, No. 2, 2000, pp. 999-1005. doi:10.1006/jmaa.2000.6991

[7] F. Riesz and B. Sz.-Nagy, "Functional Analysis,” Fredrick Ungar Publishing Co., New York, 1955.

[8] I. Husain and S. K. Srivastav, "On Nondifferentiable Nonlinear Programming,” Journal of Applied Mathematics and Bioinformatics, Vol. 3, No. 2, 2013, pp. 45-64.

[9] V. Chankong and Y. Y. Haimes, "Multiobjective Decision Making Theory and Methodology,” North-Holland, New York, 1983. 